\title{
Enhanced photocatalytic properties in well-ordered mesoporous $\mathrm{WO}_{3}^{\dagger}$
}

\author{
Li Li, ${ }^{a}$ Methira Krissanasaeranee, ${ }^{b}$ Sebastian W. Pattinson, ${ }^{b}$ Morgan Stefik, ${ }^{c}$ \\ Ulrich Wiesner, ${ }^{c}$ Ullrich Steiner ${ }^{* a}$ and Dominik Eder $* b$
}

Received 4th May 2010, Accepted 31st August 2010

DOI: $10.1039 / \mathrm{c0cc01237h}$

We used polyisoprene-block-ethyleneoxide copolymers as structure-directing agents to synthesise well-ordered and highly-crystalline mesoporous $\mathrm{WO}_{3}$ architectures that possess improved photocatalytic properties due to enhanced dye-adsorption in absence of diffusion limitation.

As a green technology, semiconductor photocatalysis has attracted increasing interest driven by the search for new energy sources during the past few decades. ${ }^{1}$ The performance of the photocatalysts strongly depends on their crystal structure and morphology. ${ }^{1,2}$ It is widely recognized that high crystallinity and a continuous network architecture with controlled pore sizes that facilitate molecular access to high surface areas are highly desired for maximizing their photocatalytic performance. ${ }^{3,4}$ This has recently stimulated intensive research in designing mesoporous metal oxides, such as $\mathrm{TiO}_{2}, \mathrm{Nb}_{2} \mathrm{O}_{5}$, and $\mathrm{ZrO}_{2}{ }^{5}$ The major drawback of these attempts is that the oxide structures often contain a significant amount of undesired amorphous content, which facilitates the recombination of electrons and holes, thereby limiting the catalytic efficiency. ${ }^{2}$ The crystallinity can be improved by annealing at high temperatures, upon which, however, the ordered structure typically collapses. Hence, synthesising well-ordered mesoporous structures with high crystallinity still remains a major challenge. ${ }^{6}$

Recently, we used polyisoprene-block-ethyleneoxide (PI- $b$-PEO) copolymers as structure-directing agents to synthesise $\mathrm{TiO}_{2}$ with controlled mesoporous structures. ${ }^{7,8}$ The large interaction parameter between the PI and PEO blocks and the high degree of polymerization allows the rapid formation of structures with long-range order. In addition, the PI- $b$-PEO morphology has relatively large pore sizes, which facilitate the effective infiltration of functional materials. The resulting $\mathrm{TiO}_{2}$ exhibited excellent performance in dye-sensitized solar cells. Although $\mathrm{TiO}_{2}$ is currently the most studied semiconductor photocatalyst, its wide band gap $(3.2 \mathrm{eV})$ limits $\mathrm{TiO}_{2}$ to a small ultraviolet fraction of solar energy. ${ }^{9}$ In contrast, tungsten trioxide $\left(\mathrm{WO}_{3}\right)$ has a narrower band gap $(2.4-2.8 \mathrm{eV})$ that enables harvesting visible light. ${ }^{10}$ Only a few studies have so far been devoted to the synthesis of porous $\mathrm{WO}_{3}$, i.e. using PMMA spheres ${ }^{11}$ and by anodization of

${ }^{a}$ Cavendish Laboratory, University of Cambridge,

J. J. Thomson Avenue, Cambridge, CB3 OHE, UK.

E-mail: u.steiner@phy.cam.ac.uk

${ }^{b}$ Department of Materials Science \& Metallurgy,

University of Cambridge, Cambridge, CB2 3QZ, UK.

E-mail:de235@cam.ac.uk

Department of Materials Science and Engineering,

Cornell University, Ithaca, New York, 14853, USA.

† Electronic supplementary information (ESI) available: Additional

SEM images, pore size distribution and photocatalytic data. See DOI:

$10.1039 / \mathrm{c} 0 \mathrm{cc} 01273 \mathrm{~h}$ tungsten foil. ${ }^{12}$ Despite yielding only macroporous and weakly-ordered structures these studies still demonstrated enhanced photocatalytic properties compared with their dense counterparts. It is therefore likely that improving the pore structure and crystallinity will maximise the photocatalytic performance of $\mathrm{WO}_{3}$. Herein, we demonstrate the synthesis of highly-crystalline mesoporous $\mathrm{WO}_{3}$ with well-ordered pore architectures using a sol-gel process and PI- $b$-PEO copolymers as structure-directing agents. We investigated the effect of polymer-to- $\mathrm{WO}_{3}$ weight ratio on the morphology and tested the photocatalytic performance for the degradation of methylene blue under visible light.

In a typical synthesis, $45 \mathrm{mg}$ PI- $b$ - $\mathrm{PEO}^{13}$ block copolymer $\left(M_{\mathrm{n}}=37.3 \mathrm{~kg} \mathrm{~mol}^{-1}, 27 \mathrm{wt} \%\right.$ PEO) was dissolved in $1.2 \mathrm{ml}$ anhydrous chloroform. The polymer solution was then added

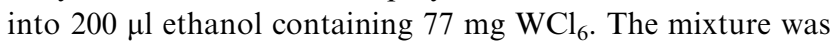
vigorously stirred for 4 hours and then left to gel overnight in an open Petri dish at $70{ }^{\circ} \mathrm{C}$. The as-made bulk samples were calcined in air at $400{ }^{\circ} \mathrm{C}$ for 4 hours, which induced crystallisation and at the same time removed the polymer template. The polymer-to- $\mathrm{WO}_{3}$ weight ratio was varied between $0: 1$ and $1.67: 1$ to investigate the effect of the polymer content on the resulting morphology.

Fig. 1 shows typical SEM (LEO $1530 \mathrm{VP}$ ) and TEM (JEOL 200FX) images of $\mathrm{WO}_{3}$ samples synthesized with various

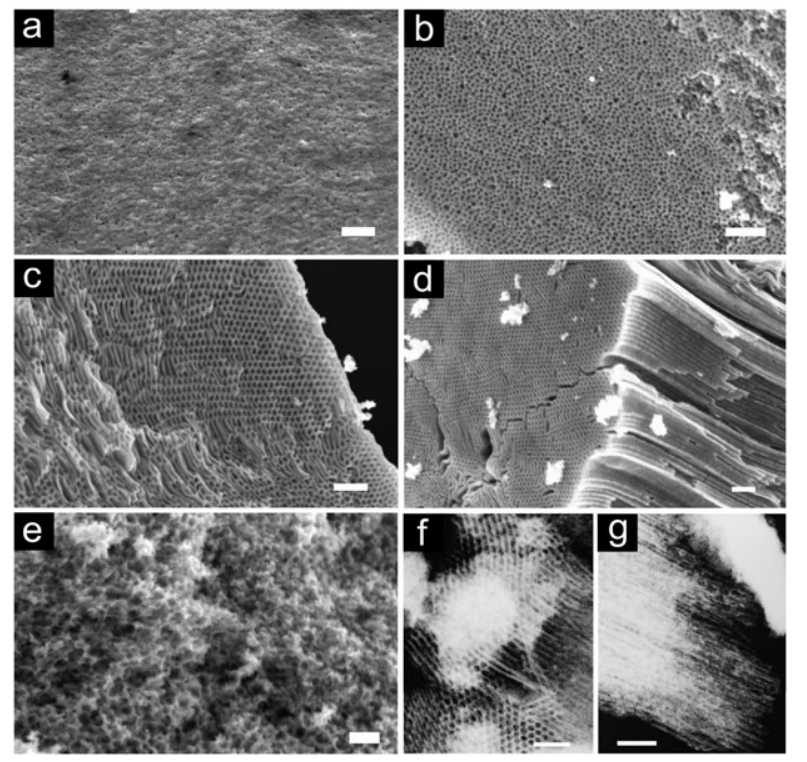

Fig. 1 SEM images of $\mathrm{WO}_{3}$ synthesized with various polymer : $\mathrm{WO}_{3}$ weight ratios (a) $0: 1$, (b) $0.17: 1$, (c) $1: 1$, (d) $1.25: 1$, and (e) $1.67: 1$ after calcination at $400{ }^{\circ} \mathrm{C}$. TEM (f-g) images of sample with ratio $1: 1$ after photocatalysis showing preserved porous structure. Scale bar: $200 \mathrm{~nm}$. 
polymer-to- $\mathrm{WO}_{3}$ weight ratios. In absence of the polymer (Fig. 1a) the sample consisted of densely-packed agglomerates of particles with sizes between 25-30 nm. In contrast, the addition of polymer resulted in porous structures. For instance, a ratio of $0.17: 1$ (Fig. 1b) gave rise to micellar mesopores of uniform diameter $(10-13 \mathrm{~nm})$. The pore size increased with increasing polymer content to approximately 20-25 nm for the $1: 1$ ratio. At the same time, the wall thickness of $\mathrm{WO}_{3}$ remained almost unchanged (10-15 nm). It is important to note that along with the pore diameters also the porous architecture can be effectively controlled by the polymer content. For instance, for ratios below $1: 1$, the structure is micellar in nature, while ratios between $1: 1$ and $1.25: 1$ yielded vertically aligned, cylindrical mesopores with lengths similar to the film thickness (Fig. 1c-d); only a small fraction of micellar pores was found near fractures and edges. As the polymer content was further increased to $1.67: 1$, the ordered structure collapsed, indicating that the $\mathrm{WO}_{3}$ content was likely not sufficient to mechanically support the ensuing porous network (Fig. 1e).

The porosity and specific surface area were determined by nitrogen physisorption according to Barret-Joyner-Halenda $(\mathrm{BJH})$ and Brunauer-Emmett-Teller (BET, Tristar), respectively. The BET isotherms show Type IV behaviour with H1-hysteresis, which is typical for well-ordered materials. Fig. 2a shows the pore size distributions for the samples synthesised with various polymer-to- $\mathrm{WO}_{3}$ ratios. With the exception of the reference sample (0:1 ratio), all templated samples show a sharp peak in the mesoporous regime, indicating a narrow pore size distribution. The average pore size increased from $9 \mathrm{~nm}$ to $23 \mathrm{~nm}$ for $0.17: 1$ and $1: 1$ ratios, respectively, which agrees well with the SEM observations. Starting from a value of $32.5 \mathrm{~m}^{2} \mathrm{~g}^{-1}$ for the reference sample, the surface area increased with increasing polymer content to a maximum of $55.5 \mathrm{~m}^{2} \mathrm{~g}^{-1}$ for the $1: 1$ ratio. The sample with the highest $1.67: 1$ ratio showed a broader pore size distribution and yielded a slightly lower surface area $\left(48.2 \mathrm{~m}^{2} \mathrm{~g}^{-1}\right)$ compared to the $1: 1$ sample. This is in agreement with a partially collapsed porous network.

Wide-angle X-ray diffraction (XRD, Bruker D8 Advanced) confirms that all samples are crystalline and of monoclinic phase (Fig. 2b). The crystal size was calculated from Scherrer's equation using the (111) diffraction peak at $28.8^{\circ}$. Interestingly, the crystal sizes of all mesoporous samples are similar $(\sim 12 \mathrm{~nm})$ to the reference sample. It appears that the formation of $\mathrm{WO}_{3}$ crystals was not noticeably altered by the confinement imposed by the block copolymer. The combined results
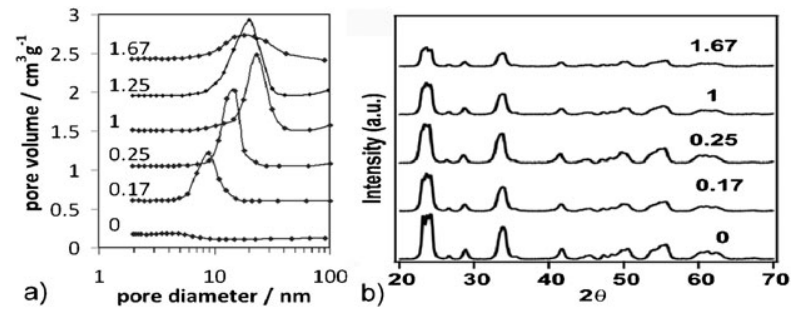

Fig. 2 (a) Pore size distributions obtained from $\mathrm{N}_{2}$ desorption and (b) X-ray diffraction patterns of $\mathrm{WO}_{3}$ samples (JCPDS: 20-1324) synthesized with various polymer-to- $\mathrm{WO}_{3}$ weight ratios after calcination at $400{ }^{\circ} \mathrm{C}$. indicate the simultaneous achievement of ordered mesostructure and crystallinity by our protocol.

The photocatalytic properties of the $\mathrm{WO}_{3}$ samples were evaluated for the photodegradation of methylene blue (MB) under visible light. Typically, $10 \mathrm{mg}$ of the $\mathrm{WO}_{3}$ sample was dispersed in $50 \mathrm{ml}$ of distilled water containing $4.8 \mathrm{ppm} \mathrm{MB}$. The suspension was stirred in the dark for 2 hours to ensure equilibrium of the dye adsorption on the surface of the photocatalysts, after which the samples were exposed to visible light $(72 \mathrm{~W}, \mathrm{UV}$ cut-off at $\sim 420 \mathrm{~nm})$. An aliquot part of the solution $(\sim 1 \mathrm{ml})$ was taken in regular intervals and centrifuged for $2 \mathrm{~min}$ to separate the solution from catalyst residues. The MB concentration was quantified by UV-Vis spectroscopy (Perkin Elmer, Lambda 850), following the absorption maximum at $\lambda=664 \mathrm{~nm}$. It is important to note that the micellar and cylindrical pore structures of the catalysts were not changed in any way by the photocatalytic testing (Fig. 1e-g and ESI $\dagger$ ).

The initial concentration of MB decreased noticeably upon equilibration in the dark. As a result of their larger surface area the mesoporous samples adsorbed considerably more dye molecules (i.e. $67 \%$ for the $1: 1$ ratio) than the reference $(31 \%)$. Taking $1.97 \mathrm{~nm}^{2}$ as the unit area of adsorbed $\mathrm{MB},{ }^{14}$ the adsorbed quantities correspond to surface coverages of $\sim 75 \%$ for the reference and $>97 \%$ for the mesoporous samples. Consequently, the entire surface area of the mesoporous samples is accessible, while the adsorption of $\mathrm{MB}$ in the reference may be blocked by agglomerated $\mathrm{WO}_{3}$ particles resulting in pores smaller than $2.6 \mathrm{~nm}$, the minimum diameter allowing the passage of MB molecules. ${ }^{15}$

Fig. 3a presents the decrease of $\mathrm{MB}$ concentration vs. time for the different $\mathrm{WO}_{3}$ samples under visible light, normalised for the equilibrated $\mathrm{MB}$ concentration. In absence of a catalyst the $\mathrm{MB}$ concentration remains stable throughout the entire reaction, providing a solid base line. All mesoporous samples degraded MB considerably faster than the reference, hence exhibiting higher photocatalytic activities. For instance, the conversion of $\mathrm{MB}$ after 5 hours of irradiation using the reference was only $18 \%$, while $53 \%$ of MB was degraded by the sample with $1: 1$ ratio (Fig. $3 b$ ). This amounts to an almost three-fold increase in activity. Considering that the corresponding specific surface area of the mesoporous sample was increased by only $70 \%$, it is likely that any restrictions imposed on the transport of reactant molecules by the small pores in the reference sample is significantly reduced in the mesopores. Furthermore, we analysed a highly-dispersed commercial $\mathrm{WO}_{3}$ nanopowder (Sigma-Aldrich) and observed
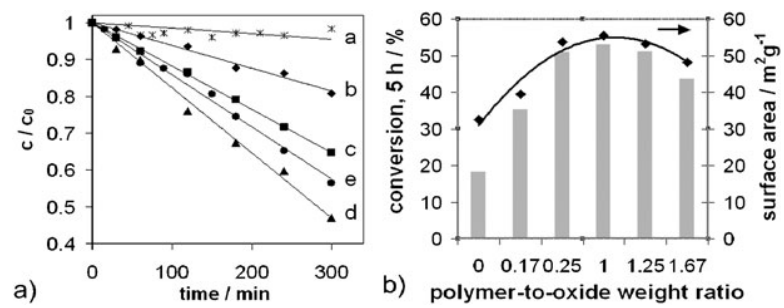

Fig. 3 (a) Decrease in absorbance of MB over time (a) no catalyst, (b-e) for $\mathrm{WO}_{3}$ with ratios $0,0.17,1,1.67: 1$; (b) conversion after 5 hours reaction time in visible-light and surface area $v s$. weight ratios. 
a similar activity compared with the mesoporous sample (ESI $\dagger$ ), thus confirming that diffusion limitations in our mesoporous samples were indeed effectively eliminated.

To identify the reactant molecule that is most strongly affected by diffusion limitation, we need to consider the photocatalytic degradation pathway of MB in water (ESI $\dagger$ ). According to Houas et al., ${ }^{15}$ the MB molecule adsorbs perpendicularly to the surface of the photocatalyst via its cationic sulfur group, which is then oxidised to the sulfoxide via an electrophilic attack of $\mathrm{OH}^{*}$, formed upon oxidation of water by photo-produced holes in the photocatalyst. This induces the opening of the central aromatic ring in order to conserve the double bond conjugation. The sulfoxide group immediately oxidises to sulfone, causing the definitive dissociation of the two rings. These aromatic compounds are then decomposed completely via the photo-Kolbe reaction to $\mathrm{CO}_{2}, \mathrm{NH}_{4}{ }^{+}, \mathrm{NO}_{3}{ }^{-}$and $\mathrm{SO}_{4}{ }^{2-}$ ions, which leave the pores and make way for the degradation of new MB molecules. Considering the similar sizes of the two largest molecules in this process, $\mathrm{MB}(0.72 \mathrm{~nm})$ and the sulfoxide $(0.76 \mathrm{~nm})$, as calculated from the corresponding cross-sectional areas, ${ }^{14}$ any pore size confinement will most likely affect the transport of MB itself to its adsorption site inside the pore, impeding the continuous supply of the reactant. The diffusion of liquids in pores has been studied by Ternan, who observed that even pores as large as $40 \mathrm{~nm}$ can impose serious diffusion limitations on molecules as small as $2 \mathrm{~nm} .{ }^{16}$ The effective diffusivity of molecules in pores, $D_{\text {eff, }}$ with respect to the bulk diffusivity, $D_{\mathrm{B}}$, correlates with $\lambda$, the ratio of molecule to pore radius, $r_{\mathrm{m}} / r_{\mathrm{P}}$

$$
\frac{D_{\text {eff }}}{D_{\mathrm{B}}}=\frac{(1-\lambda)^{2}}{1+P \lambda}
$$

where $P$ is a function of the solvent properties (i.e. viscosity), typically ranging from 2 to 20 . This equation can be used when the solute molecules are both sufficiently larger than the solvent molecules and sufficiently smaller than the pores. Both requirements are satisfied in the present case. The pore diameters in the mesoporous samples range from 9 to $23 \mathrm{~nm}$, as obtained from $\mathrm{BJH}$, which, using $0.72 \mathrm{~nm}$ as the lower limit for the diameter of MB, correspond to $\lambda$ values of 0.08 and 0.03 , respectively. Assuming an average value of $P=2$ typical for polar solvents such as water, ${ }^{16}$ the effective pore diffusivities are reduced by only $12 \%$ for $23 \mathrm{~nm}$ pores and $27 \%$ for $9 \mathrm{~nm}$ pores compared to the non-confined reaction. For comparison, taking an upper-limit pore size of $4 \mathrm{~nm}$ (ESI $\dagger$ ) the diffusivity in the reference sample was reduced by at least $50 \%$. In general, pore diffusivities of more than $70-80 \%$ with respect to bulk diffusivities are considered ideal. Consequently, the pore sizes of our mesoporous $\mathrm{WO}_{3}$ samples do not impose significant diffusion limitations, in contrast to the reference sample. This is further confirmed in Fig. 3b, which shows that the trend of degradation rate of MB in the mesoporous samples follows closely the observed changes in surface area. In contrast, the activity of the reference sample is significantly lower than expected from its surface area. It seems that, in absence of diffusion limitation, the photocatalytic performance of the mesoporous samples is directly controlled by the surface area and so by the number of adsorbed dye molecules. Consequently, maximising the surface area, i.e. by optimising pore size and wall thickness, will likely further improve the performance of $\mathrm{WO}_{3}$.

In summary, we used PI- $b$-PEO as a structure-directing agent for the controlled synthesis of highly crystalline $\mathrm{WO}_{3}$ photocatalysts with well-organised mesopores. These mechanically stable architectures consist either of micellar or cylindrical pores with uniform diameters and can be controlled by varying the polymer-to-inorganic ratio. These structures are excellent photocatalysts for the degradation of organic compounds as they reduce diffusion limitations of the reagents into the pores and so facilitate enhanced dye adsorption and conversion. These materials may also be of interest for use as supports for noble metal catalysts, in electrochromic devices, and as model systems for studying pore transport processes in metal oxides.

We acknowledge the Nokia Research Centre, the European Research Council (EP/F056702/1, and Advanced Investigator award to A. K. Cheetham), and the Cornell Universiy KAUST Center for Research and Education for funding. We thank Stefan Guldin for valuable discussions.

\section{Notes and references}

1 A. Mills and S. L. Hunte, J. Photochem. Photobiol., A, 1997, 108, 1.

2 B. Ohtani, Y. Ogawa and S. Nishimoto, J. Phys. Chem. B, 1997, 101, 3746.

3 J. Tang, Y. Y. Wu, E. W. McFarland and G. D. Stucky, Chem. Commun., 2004, 1670.

4 C.-K. Tsung, J. Fan, N. Zheng, Q. Shi, A. J. Forman, J. Wang and G. D. Stucky, Angew. Chem., Int. Ed., 2008, 47, 8682.

5 P. Yang, D. Zhao, D. I. Margolese, B. F. Chmelka and G. D. Stucky, Nature, 1998, 396, 152.

6 J. Lee, M. C. Orilall, S. C. Warren, M. Kamperman, F. J. Disalvo and U. Wiesner, Nat. Mater., 2008, 7, 222.

7 M. Nedelcu, J. Lee, E. J. W. Crossland, S. C. Warren, M. C. Orilall, S. Guldin, S. Huttner, C. Ducati, D. Eder, U. Wiesner, U. Steiner and H. J. Snaith, Soft Matter, 2009, 5, 134.

8 M. Nedelcu, S. Guldin, M. C. Orilall, J. Lee, S. Huttner, E. J. W. Crossland, S. C. Warren, C. Ducati, P. R. Laity, D. Eder, U. Wiesner, U. Steiner and H. J. Snaith, J. Mater. Chem., 2010, 20, 1261.

9 T. X. Wu, G. M. Liu, J. C. Zhao, H. Hidaka and N. Serpone, J. Phys. Chem. B, 1998, 102, 5845.

10 G. R. Bamwenda and H. Arakawa, Appl. Catal., A, 2001, 210, 181.

11 M. Sadakane, K. Sasaki, H. Kunioku, B. Ohtani, W. Ueda and R. Abe, Chem. Commun., 2008, 6552.

12 A. Watcharenwong, W. Chanmanee, N. R. de Tacconi, C. R. Chenthamarakshan, P. Kajitvichyanukul and K. Rajeshwar, J. Electroanal. Chem., 2008, 612, 112.

13 J. Allgaier, A. Poppe, L. Willner and D. Richter, Macromolecules, 1997, 30, 1582.

14 D. Graham, J. Phys. Chem., 1955, 59, 896.

15 A. Houas, H. Lachheb, M. Ksibi, E. Elaloui, C. Guillard and J.-M. Herrmann, Appl. Catal., B, 2001, 31, 145.

16 M. Ternan, Can. J. Chem. Eng., 1987, 65, 244. 